\title{
The sex Differences in Blood Pressure and Cardiac Autonomic Modulation during the Menopause Transition at Rest and after Aerobic Exercise Training
}

\author{
Maycon Jr Ferreira1,2, Rodrigo D Esposti1, Aline O Jarrete1,3, Carlos H G Sponton1,4, \\ Angelina Zanesco1,5 \\ ¿Laboratory of Cardiovascular Physiology and Exercise Science, Institute of Biosciences, \\ UNESP, Rio Claro, Brazil. \\ ${ }_{2}$ Physiology Exercise Laboratory, Department of Physiology, Federal University of São \\ Paulo, São Paulo, SP, Brasil. \\ 3Department of Structural and Functional Biology, Institute of Biology, UNICAMP- \\ University of Campinas, Campinas, SP, Brazil. \\ 4Department of Cell and Tissue Biology, University of California, San Francisco, CA, USA. \\ sMetropolitan University of Santos (UNIMES), Santos, SP, Brazil.
}

Running title: Menopausal transition and cardiovascular system

Conflict of Interest/Disclosure: None.

Corresponding author:

Angelina Zanesco, Professor in Physiology

Metropolitan University of Santos

Avenida Conselheiro Nébias n ${ }^{\circ} 536$ - Encruzilhada

Santos/SP

Tel: +55 19997512416

Email: angelina.zanesco@gmail.com 


\section{ABSTRACT}

Background: Sex differences in blood pressure (BP) exist during all reproductive life between women and men whereas a sharper increase in BP occurs after menopause which is associated with increased prevalence of cardiovascular diseases. This study examined cardiovascular and biochemical parameters in perimenopausal women (PW) aiming to investigate the influence of sex on a) office BP and for 24 hours; b) cardiac autonomic modulation; c) redox state by measuring MDA, SOD, and catalase; d) NOx-concentration. In addition, aerobic exercise training (AET) was applied for detecting changes in cardiovascular responsiveness during the menopausal transition. Methods: Thirty-one participants were enrolled, healthy PW and age-matched men. Cardiovascular and biochemical biomarkers were evaluated at baseline and after AET (8 weeks of exercise on a treadmill, three sessions/week, duration of 30-40 minutes).

Results: At rest, PW presented: a) a lower diastolic BP during daytime; b) a lower absolute and normalized LF component as well as a higher HF normalized component; d) no sex differences for redox biomarkers and $\mathrm{NO}_{x-}$ concentration. After AET, only PW were responsive in lowering systolic BP that was accompanied by an increase in $\mathrm{NO}_{\mathrm{x}-}$ concentration and SOD activity. Regarding HRV, both groups were responsive to the AET.

Conclusions: During the menopausal transition, systolic BP was similar to men whereas cardiac autonomic modulation remained unaltered showing the influence of sex steroids on BP. In Addition, AET was fundamental during the menopause transition by preventing an elevation in $\mathrm{BP}$, minimizing the effects of aging associated with estrogen deficiency on women's cardiovascular health.

Keywords: sex differences, blood pressure, redox state, perimenopausal women, nitric oxide 
The existence of sex differences related to blood pressure (BP) levels is well documented. On the other hand, a sharper increase in BP in women than in men has been reported during midlife which is associated with increased prevalence of cardiovascular diseases after menopause (Barton \& Meyer, 2009; Benjamin et al., 2018). Estrogen deficiency is considered the main cause of the deep increase in BP after menopause since estrogen plays a key role in the regulation of a variety of physiological systems including cardiovascular, endocrine, nervous and immune (Stork et al., 2004). Nevertheless, most of the data reporting the role of estrogen deficiency on BP levels are from experimental studies (Reckelhoff, 2018). Therefore, studies with women in different phases of the climacteric period are fundamental to understand the possible mechanisms as well as biomarkers that are involved in BP regulation in this particular population. Indeed, many questions still unanswered related to women and cardiovascular health. Is there an endothelial dysfunction after menopause? Previous studies have systematically shown that NO/cGMP signaling pathway, as well as flow-mediated dilation, is not impaired in hypertensive postmenopausal women as compared with normotensive subjects (Sponton et al., 2014; Puga et al., 2016; Jarrete et al., 2016; Novais et al., 2017). Additionally, both hypertensive and normotensive postmenopausal women were responsive to aerobic exercise training (AET) by increasing the endothelial biomarkers related to NO/cGMP pathway (Sponton et al., 2010; Rezende et al., 2011). Another question in this scenario that should be raised is: when is the turning point of the increasing BP during the climacteric period? Thus, the objective of this study was to examine the cardiovascular and biochemical parameters in perimenopausal women compared with age-matched men. This period is crucial for women's health since sex hormones fluctuate from month to month and this intermittent hormonal exposure might be involved in the cardiovascular effects of the 
postmenopausal women where a complete decline in ovarian function and estrogen levels takes place. To address this issue, we examine the influence of sex on a) office BP and during 24 hours; b) autonomic modulation by evaluating heart rate (HR) and its variability (HRV); c) redox state by measuring biomarkers (MDA, SOD and catalase); d) nitric oxide (NO) production by measurement of $\mathrm{NO}_{\mathrm{x}}$ - concentration. In addition, AET was applied as a challenge for detecting cardiovascular responsiveness during the perimenopausal period.

\section{Materials and methods}

\section{Participants}

This study was approved by the Ethical Committee from University of Sao Paulo State (Protocol: 908.577/2015). Disclosure and participants recruitment were performed by advertisement in the surrounding area of the university and local media. Participants were included according to the following criteria: age between 40 and 59 years old, inactive physically $(<150$ minutes of moderate physical activity or $<75$ minutes of vigorous physical activity), non-obese , nonsmoking, non-prediabetes (fasting blood glucose $<100 \mathrm{mg} / \mathrm{dL}$ ), being in the perimenopausal period (exclusive for women), normal renal function (creatinine levels $<135 \mathrm{mmol} / \mathrm{L}$ ), nonalcoholic (<3 cups/day), no menopausal therapy (exclusive for women), no use of antihypertensive medications, any other diseases that would prevent the practice of physical exercise. After recruitment, all participants $(n=31)$ underwent a general anamnesis followed by the fulfillment of questionnaires related to the practice of physical activity (PAR-Q) and quality of life (SF-36). Participants were invited to sign a free consent term.

The period of evaluation lasted approximately $3-4$ weeks and $1-2$ weeks, before and after of the AET, respectively. At the beginning of the study, all the participants performed a familiarization period in a treadmill (Treadmill RT 250 PRO) that consisted of walking during 4 - 
6 sessions of 20 minutes each. The aims were to teach and to guide them on how to walk in a treadmill correctly as well as to avoid any stressful situation that would interfere with the measurements.

Peak oxygen consumption was determined indirectly in a treadmill by performing the 1mile test according to Kline et al. (1987). Maximal Lactate Steady State (MLSS) test was performed to determine the optimal walking intensity of each volunteer for AET. After that, the participants exercised on a treadmill, three sessions/week, totalizing twenty-four sessions of physical exercise as previously described (Ferreira et al., 2018).

The perimenopause characterization in women was performed through by self-report of irregular menstrual cycles and the presence of signs and symptoms of this period, as previously described by Harlow et al. (2012). In addition, follicle-stimulating hormone (FSH), luteinizing hormone (LH) and 17ß-estradiol were measured.

\section{Anthropometric and cardiovascular parameters}

Body weight and height of the participants were measured on a digital scale (Toledo® 2096 PP). BMI was calculated by the ratio of body weight/height ${ }^{2}$. Waist circumference measurements were performed in triplicate between the iliac crest and the last rib.

The measurements of the resting heart rate (HR) and heart rate variability (HRV) were carried out in a quiet room, noise-free and environment-controlled temperature. The subjects remained in absolute rest in the sitting position for 15 minutes, comfortably, maintaining a constant respiratory rate. Values were recorded and stored through of a transmitter belt placed at the level of the distal third of xiphoid process with a pulse HR monitor (Polar RS800CX, Electro Oy, Kempele, Finland) as previously described by Barbosa et al. (2016). The participants were instructed to maintain their normal diet and not to consume any type of drink and/or make use of 
stimulant medication that could interfere in both parameters. In addition, they were instructed not to engage in any vigorous exercise in the last 48 hours preceding the test. Subsequent to the data acquisition, the records were transferred to Polar ProTrainer $5^{\mathrm{TM}}$ software. Resting HR was calculated as the mean value of HR during the recording period.

The HRV measurement was performed through the analysis of the normal intervals RR (iRR). The selection of the section for analysis was performed by visual inspection of the distribution of the iRR in the interval of fifteen minutes, in which a central five minutes interval of greater stability of the signal was selected, containing a sampling frequency of, at least, 256 points. The remaining ten minutes, distributed between initial and final passages, were discarded. The iRR analysis was performed using Kubios HRV 2.1 software (University of Eastern Finland, Kuopio, Finland). The HRV measurement was performed using the indices corresponding to the frequency domain. For this analysis, the indices selected were the power in the low-frequency range (LF), and in the high-frequency range (HF), (Kleiger et al., 2005). The HF component is defined as a vagal modulating marker which is synchronized with the respiratory rate . Both powers were calculated in absolute $\left(\mathrm{ms}^{2}\right)$ and normalized units (nu). In addition, the variance of all successive normal iRRs (TP) and the ratio between low- and high-frequency (LF/HF) were used as an index of sympathovagal balance (Task Force, 1996).

Office BP and Ambulatory BP monitoring (ABPM) were obtained in accordance with international guidelines as described previously (Sponton et al., 2014).

\section{Biochemical analyses}

Blood samples were collected after a 12-hour overnight fast. Samples were centrifuged and the supernatant (plasma or serum) was collected and stored in aliquots at $-80^{\circ}$ for biochemical 
analyses. A specific commercial kit (Cayman Chemical, Ann Arbor, MI, USA) was used for all the tests.

FSH, LH, and 17- $\beta$ estradiol: measured by using the sandwich-type immunometric method (ELISA) (Cayman Chemical, Ann Arbor, MI, USA) in accordance to the manufacturer. The analysis of 17- $\beta$ estradiol was performed in a private clinic.

The endogenous production of nitric oxide (NO): quantified indirectly through the nitrite and nitrate anions (NOx-) measurements, as previously described by Delbin et al. (2012).

Lipid peroxidation: quantified based on the serum concentrations of the compound malondialdehyde (MDA).

Superoxide dismutase (SOD) activity: determined using a tetrazolium salt for detection of superoxide radicals generated by xanthine oxidase and hypoxanthine.

Catalase: the method of determining the activity of catalase occurred through its activity peroxidase.

\section{Statistical analysis}

Data are presented as means \pm standard deviation (SD). The normality of the data was verified by the Kolmogorov-Smirnov test. After that, the effects of sex, AET and sex x training interaction were obtained through the analysis of variance (ANOVA) of repeated measures adjusted for two factors (sex and training), followed by Bonferroni post-hoc when appropriate. Differences in the magnitude of behavior ambulatory BP reduction of 24 hours were verified through analysis of the area under the curve (AUC), using a paired $t$-test for comparison before and after AET in each group, and an unpaired $t$-test for comparison between groups. Analyzes were performed using SPSS statistical software for Windows (PASW Statistics 18). $\mathrm{P}<0.05$ was considered as statistically significant. 


\section{Results}

The general characteristics of the participants are shown in Table 1. At the baseline, the studied population was similar in age and BMI. On the other hand, men showed higher weight waist circumference as compared with perimenopausal women, $([\mathrm{F}(1,29)=11.48 ; \mathrm{p}<0.002]$ and $\left[\mathrm{F}_{(1,29)}=14.88 ; \mathrm{p}<0.001\right]$, respectively). As expected, the $\mathrm{VO}_{2}$ peak values were higher for men compared with women $\left(\left[\mathrm{F}_{(1,29)}=48.07 ; \mathrm{p}<0.0001\right]\right)$. In contrast, HR was lower in men than women $([\mathrm{F}(1,29)=8.93 ; \mathrm{p}<0.007])$. Daytime diastolic ABPM $([\mathrm{F}(1,29)=12.23 ; \mathrm{p}<0.003])$ and 24 hours diastolic ABPM $\left(\left[\mathrm{F}_{(1,29)}=12.43 ; \mathrm{p}<0.001\right]\right)$ were lower in perimenopausal women compared with men. In addition, HRV showed a higher absolute $L F$ component in men $([F(1,29)=7.68 ; p<0.02])$. A higher normalized LF $\left(\left[\mathrm{F}_{(1,29)}=5.74 ;<0.03\right)\right.$ and a lower HF component only before AET, $([\mathrm{F}(1,29)=5.66 ; \mathrm{p}<0.03])$ were observed in men as compared with women.

Twenty-four sessions of AET promoted a decrease in weight $(-1.4 \%)([\mathrm{F}(1,29)=7.09$;

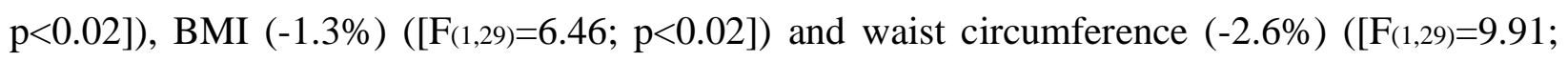
$\mathrm{p}<0.005])$ only in men. On the other hand, both groups showed an increase in the aerobic capacity (2.1 and $5 \%$ for men and women, respectively) $([\mathrm{F}(1,29)=17.25 ; \mathrm{p}<0.001)$. In addition, no differences were observed for chronotropic response in both trained groups as measured by mean resting HR (Table 1). Examining HRV in the frequency domain, we found that AET promoted a decrease in absolute LF component only in men group $(-53.0 \%)([\mathrm{F}(1,29)=17.32 ; \mathrm{p}<0.001])$ whereas normalized LF component was decreased $\left(\left[\mathrm{F}_{(1,29)}=45.19 ; \mathrm{p}<0.001\right]\right)$ in both groups $(-24.8$ and $28.6 \%$ for men and perimenopausal women, respectively). Similarly, absolute $([\mathrm{F}(1,29)=7.53$; $\mathrm{p}<0.02])$ and normalized HF component $([\mathrm{F}(1,29)=45.38 ; \mathrm{p}<0.001])$ were increased in response to AET in men (81.6 and $166.3 \%$, respectively) while only normalized HF component was increased in women $(100.3 \%)$. The $\mathrm{LF} / \mathrm{HF}$ ratio was significantly decreased $\left(\left[\mathrm{F}_{(1,29)}=28.30 ; \mathrm{p}<0.001\right]\right)$ in 
men $(-72.6 \%)$ and perimenopausal women group (-71.6\%) after AET. Data are summarized in Table 1.

Regarding office BP measurements, only perimenopausal women were responsive to AET, showing a decrease of $-5.6 \%$ for systolic office $\mathrm{BP}([\mathrm{F}(1,29)=17.41 ; \mathrm{p}<0.001])$. Additionally, no difference was found for diastolic office BP in both trained groups (Table 1). In agreement with that, trained perimenopausal women presented a decrease in systolic BP during nighttime as measured by ABPM $(-4.2 \%)\left(\left[\mathrm{F}_{(1,29)}=5.09 ; \mathrm{p}<0.04\right]\right)$ as well as for systolic BP for 24 hours (approximately $-3.0 \%)([\mathrm{F}(1,29)=5.64 ; \mathrm{p}<0.03])($ Figure 1$)$.

Anova repeated measure showed that there was an interaction for NOx-concentration $([\mathrm{F}(1,29)=5.49 ; \mathrm{p}<0.05])$. Bonferroni post-hoc showed a significant increase in the NOxconcentration $(38.5 \%)$, a biomarker related to NO production, in trained perimenopausal women but no changes were seen in trained men. Regarding redox status, SOD activity was significantly increased $(55.1 \%)$ in response to AET only in perimenopausal women $([\mathrm{F}(1,29)=8.67 ; \mathrm{p}<0.007])$ but neither MDA concentrations nor catalase activity was affected by exercise training in both groups.

\section{Discussion}

Comparing to age-matched men and prior to the challenge of the AET, our study shows that perimenopausal women present: a) a lower diastolic BP during daytime $([\mathrm{F}(1,29)=12.23$;

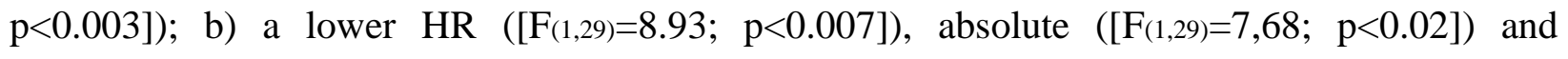
normalized LF component $([\mathrm{F}(1,29)=5.74 ;<0.03])$; and a higher HF normalized component $[F(1,29)=5.66 ; p<0.03] ; c)$ no sex differences for redox biomarkers (MDA levels, SOD and catalase

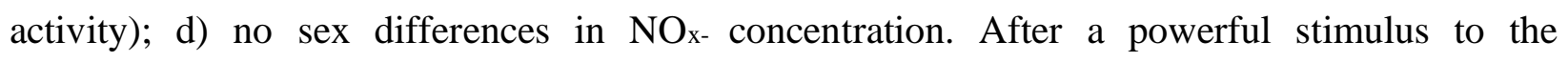
cardiovascular system provided by 24 sessions of AET, perimenopausal women were responsive in lowering systolic $\mathrm{BP}$ in office and for $24 \mathrm{~h}$ that was accompanied by increased $\mathrm{NO}_{\mathrm{x}-}$ 
concentration and SOD activity even though men were more responsive in reducing body weight and waist circumference. Regarding HRV, both groups were responsive to the AET with a reduction in LF component and increment in the HF component.

\section{Sex differences at baseline}

The perimenopause comprises the period in which the first symptoms of ovarian aging begin, indicating the approach of the menopause, until the first year after the last menstruation, occurring on average at forty-seven years of age regardless of the obesity, race/ethnicity, and smoking (Randolph et al. 2011). In this period occurs several changes in sex hormones with an increase in FSH and a decrease in estradiol levels (Burger et al., 1999). Estrogen deficiency has been pointed out as the main cause for the development of cardiovascular diseases after menopause, mainly arterial hypertension (Wassertheil-Smoller et al., 2000; Stork et al., 2004). Nevertheless, it is not well clear when the turning point of the increasing BP takes place during the climacteric period. Our findings revealed that office BP values were similar between the men and women indicating a change in BP behavior during this particular period of the women's lives. BP monitoring for 24 hours showed that only diastolic BP (daytime) was lower as compared with men. These findings show that during the menopausal transition, the sex differences in BP regulation is altered and neither redox state, measured by MDA and antioxidant enzymes activity,

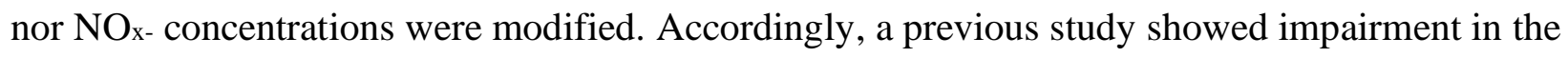
brachial artery flow-mediated dilatation during the perimenopausal transition as compared with the beginning of this period (early perimenopause), they did not measure any biomarkers though (Moreau et al., 2012). Another study found morphological alterations in common carotid artery during the menopausal transition that was associated with a decline in estrogen level (El Khoudary et al., 2013). Collectively, our data show that the sex difference in BP levels is significantly 
reduced during the menopausal transition, apparently without a contribution of the vasodilator agent, NO, measured by its bioavailability. One possibility to explain this change in BP behavior during menopausal transition could be an unbalance between vasodilation and vasoconstriction responses in the vasculature. Indeed, a previous study demonstrated that intracoronary infusion of $17 \beta$-estradiol to postmenopausal women promoted a decrease in endothelin-1, a potent vasoconstrictor agent derived from endothelial cells. Moreover, the infusion of $17 \beta$-estradiol did not affect the $\mathrm{NO}_{\mathrm{x}-}$ concentrations in this population (Webb et al., 2000).

Regarding HRV, our results demonstrate a vagal predominance in perimenopausal women compared with age-matched men showing a normal sympathovagal balance in this population even though they are under an intermittent sex hormonal exposure. Previous studies have shown a reduction in the parasympathetic component in women after menopause (Eaker et al., 1993; Kuo et al., 1999; Evans et al., 2001). In contrast, other studies show no difference in resting cardiac autonomic modulation between perimenopausal and postmenopausal women (MyslivecEk et al., 2002) or any changes in cardiac autonomic activity evaluated by 24 -hour indices of HRV after estrogen administration for 3 months in healthy postmenopausal women (Fernandes et al., 2005). Accordingly, our study clearly shows that cardiac autonomic modulation is unchanged in perimenopausal women reinforcing that sex hormone does not affect HRV. Nevertheless, it is important to emphasize that more studies with a large number of participants would bring more robust data.

As expected, lower aerobic capacity was observed in perimenopausal women, measured through the $\mathrm{VO}_{2}$ peak, compared with men, corroborating with previous studies (Fleg et al., 2005; Jackson et al., 2009; Wang et al., 2010). 
Our findings show that the volume and the intensity of the AET program employed in the study were effective in increasing $\mathrm{VO}_{2}$ peak for both groups. Although the participants were composed of physically inactive individuals and they exercised at similar relative intensity, perimenopausal women presented a higher increase in $\mathrm{VO}_{2}$ peak than men. Likely, it is due to the lower aerobic capacity presented in relation to the men at the baseline, which makes them more responsive to the same physical stimulus. On the other hand, men were responsive to the AET in weight loss and in decreasing waist circumference with a consequent reduction in BMI while perimenopausal women were non-responder for anthropometric parameters to the AET. This lack of changing in perimenopausal women in response to the AET was expected since a sex difference has been described related to metabolic rate between men and women which is dependent on the amount of skeletal muscle mass as well as the density of the adrenergic receptor subtypes present in fat depot (Thompson et al. 2012).

Regarding cardiovascular parameters, perimenopausal women were responsive in lowering office (-5.6\%) and ambulatory (nighttime and 24 hours) systolic BP (approximately -4.2 and $3.0 \%$, respectively), that was accompanied by increased SOD activity and $\mathrm{NO}_{\mathrm{x}-\text { levels indicating }}$ a higher NO bioavailability to the cells in trained women. A number of studies has shown the beneficial effects of the exercise training on cardiovascular system with improvement in the relaxing responses and reduction in BP either in humans (Maeda et al., 2004; Zaros et al., 2009; Esposti et al., 2011) or in laboratory animals (Claudino et al., 2011; Delbin et al., 2012). Our findings show that endothelial cells still reactive to the shear stress induced by exercise training during menopausal transition promoting a beneficial effect on BP regulation. In contrast, neither office BP nor ABPM were altered in trained men. Moreover, men were non-responder in changing all the evaluated biomarkers after AET for 8 weeks. 
Additionally, AET promotes an increase in vagal modulation and a decrease in sympathetic modulation in both groups. Evidence has systematically shown that physical training promotes an increase in vagal modulation and a decrease in sympathetic modulation into the heart (Carter et al., 2003; Melanson \& Freedson, 2001; Kiviniemi et al., 2006). On the other hand, studies examining the effect of the exercise training on cardiac autonomic modulation in women are scarce, and no studies exist comparing this parameter during menopausal transition and men. An early study shows a similar response to exercise training, comparing perimenopausal and postmenopausal women, with a decrease in sympathetic modulation and an increase in parasympathetic modulation (MyslivecEk et al., 2002).

\section{Conclusions}

Taken together, our findings clearly show that during menopausal transition BP levels are affected by the intermittent hormonal exposure without changes vasodilator bioavailability. Fortunately, perimenopausal women still responsive to the beneficial effects of AET showing that endothelial cells are reactive to shear stress induced by physical exercise. In addition, sex differences in cardiac autonomic modulation remain unaltered with an increase in vagal modulation and a decrease in sympathetic modulation, showing no interference of sex steroids on HRV and AET enhanced these parameters. Considering that high BP and physical inactivity are considered major contributors to death and disability, the relevance of this study is unquestionable since women live longer than men, as well as, they are more susceptible for cardiovascular diseases after menopause. Therefore, actions in management BP levels by the practice of regular physical training during menopause transition is fundamental for preventing an elevation in $\mathrm{BP}$, and for 
minimizing the effects of aging associated with estrogen deficiency on women's cardiovascular health. 


\section{Funding information}

This research was supported by Sao Paulo Research Foundation (Grant number: 2001/17437-7) and a scholarship to Maycon Jr Ferreira from Coordination for the Improvement of Higher Education Personnel (CAPES).

\section{Acknowledgments}

The corresponding author, as a principal investigator, had full access to all the data in the study and takes responsibility for the integrity of the data and the accuracy of the data analysis. 


\section{References}

Barbosa, M. .P., da Silva, N.T., de Azevedo, F.M., Pastre, C.M. \& Vanderlei, L.C. (2016). Comparison of Polar ${ }^{\circledR}$ RS800G3 $3^{\mathrm{TM}}$ heart rate monitor with Polar ${ }^{\circledR}$ S810i ${ }^{\mathrm{TM}}$ and electrocardiogram to obtain the series of RR intervals and analysis of heart rate variability at rest. Clinical Physiology and Functional Imaging. 36(2),112-117.

Barton, M. \& Meyer, M.R. (2009). Postmenopausal hypertension: Mechanisms and therapy. Hypertension. 54(1),11-8.

Benjamin, E., Virani, S., Callaway, C., Chamberlain, A., Chang, A.R., Cheng, S....Muntner, P. (2018). Heart Disease and Stroke Statistics - 2018 Update. A Report from the American Heart Association. Circulation. 137(12), e67-492.

Burger, H.G., Dudley, E.C., Hopper, J.L., Groome, N., Guthrie, J.R... Dennerstein, L. (1999).

Prospectively measured levels of serum follicle-stimulating hormone, estradiol, and the dimeric inhibins during the menopausal transition in a population-based cohort of women. Journal of Clinical Endocrinology \& Metabolism. 84(11), 4025-4030.

Carter, J.B., Banister, E.W. \& Blaber, A.P. (2003). The effect of age and gender on heart rate variability after endurance training. Medicine Science Sports \& Exercise. 35(8),1333-1340.

Claudino, M.A., Delbin, M.A., Franco-Penteado, C.F., Priviero,, F.B., De Nucci, G...Zanesco, A. (2011). Exercise training ameliorates the impairment of endothelial and nitrergic corpus cavernosum responses in diabetic rats. Life Sciences. 88(5-6), 272-277.

Delbin, M.A., Davel, A.P. C., Couto, G.K., de Araújo, G.G., Rossoni, L.V...Zanesco, A. (2012). Interaction between advanced glycation end products formation and vascular responses in femoral and coronary arteries from exercised diabetic rats. PLoS One. 7(12), e53318.

Eaker, E.D., Chesebro, J.H., Sacks, F.M., Wenger, N.K., Whisnant, J.P. \& Winston, M. (1993). Cardiovascular disease in women. Circulation. 88(4 (part I)),1999-2009.

El Khoudary, S.R., Wildman, R.P, Matthews, K., Thurston, R.C., Bromberger, J.T. \& SuttonTyrrell, K. (2013). Progression rates of carotid intima-media thickness and adventitial diameter during the menopausal transition. Menopause. 20(1), 8-14.

Esposti, R.D., Sponton, C.H.G., Malagrino, P.A., Carvalho, F.C., Peres, E....Zanesco, A. (2011). Influence of eNOS gene polymorphism on cardiometabolic parameters in response to physical training in postmenopausal women. Brazilian Journal of Medical and Biological 
Research. 44(9),855-863.

Evans, J.M., Ziegler, M.G., Patwardhan, A.R., Ott, J.B., Kim, C.S...Knapp, C.F. (2001). Gender differences in autonomic cardiovascular regulation: spectral, hormonal, and hemodynamic indexes. Journal of Applied Physiology (Bethesda, Md 1985). 91(6), 2611-2618.

Fernandes, E.O., Moraes, R.S., Ferlin, E.L., Wender, M.C.O. \& Ribeiro, J.P. (2005). Hormone replacement therapy does not affect the 24-hour heart rate variability in postmenopausal women: results of a randomized, placebo-controlled trial with two regimens. Pacing Clinical Electrophysiology. 28(Suppl 1), S172-S177.

Ferreira, M. Jr. Jarrete, A.P., Esposti, R.D., Sponton, C.H.G., Anaruma, C.P. \& Zanesco, A. (2018). Evaluation of maximal lactate steady state in middle-aged hypertensive women. Motriz: Journal of Physical Education. 24 (2),e101896.

Fleg, J.L., Morrell, C.H., Bos, A.G., Brant, L.J., Talbot, L.A... Lakatta, E.G. (2005). Accelerated longitudinal decline of aerobic capacity in healthy older adults. Circulation. 112(5), 674682.

Harlow, S.D., Gass, M., Hall, J.E., Lobo, R., Maki, P... de Villers, T.J. (2012). Executive summary of the stages of reproductive aging workshop + 10: Addressing the unfinished agenda of staging reproductive aging. Menopause. 19(4),387-395.

Jackson, A.S., Sui, X., Hébert, J.R., Church, T.S. \& Blair, S.N. (2009). Role of lifestyle and aging on the longitudinal change in cardiorespiratory fitness. Archives of Internal Medicine. 169(19), 1781-1787.

Jarrete, A.P., Zanesco, A. \& Delbin, M.A. (2016). Assessment of endothelial function by flowmediated dilation in diabetic patients: effects of physical exercise. Motriz: Journal of Physical Education. 22(1), 3-11.

Kiviniemi, A.M., Hautala, A.J., Mäkikallio, T.H., Seppänen, T., Huikuri, H. V. \& Tulppo, M.P. (2006). Cardiac vagal outflow after aerobic training by analysis of high-frequency oscillation of the R-R interval. European Journal of Applied Physiology. 96(6), 686-692.

Kleiger, R.E., Stein, P.K. \& Bigger, Jr J.T. (2005). Heart rate variability: Measurement and clinical utility. Annual Noninvasive Electrocardiology.10(1),88-101.

Kline, G.M., Porcari, J.P., Hintermeister, R., Freedson, P.S., Ward, A...Rippe, J.M. (1987). Estimation of VO2 max from a one-mile track walk, gender, age, and body weight. Medicine 
Science Sports \& Exercise. 19(3),253-259.

Kuo, T.B., Lin, T., Yang, C.C., Li,C-L., Chen, C-F. \& Chou, P. (1999). Effect of aging on gender differences in neural control of heart rate. American Journal of Physiology. 277(6 (Part 2)), H2233-H2239.

Maeda, S., Tanabe, T., Otsuki, T. J., Iemitsu, M., Miyauchi, T... Matsuda, M. (2004). Moderate regular exercise increases basal production of nitric oxide in elderly women. Hypertension Research. 27(12), 947-953.

Melanson, E.L. \& Freedson, P.S. (2001). The effect of endurance training on resting heart rate variability in sedentary adult males. European Journal of Applied Physiology. 85(5),442449.

Moreau, K.L., Hildreth, K.L., Meditz, A.L., Deane, K.D. \& Kohrt, W.M. (2012). Endothelial function is impaired across the stages of the menopause transition in healthy women. Journal of Clinical Endocrinology \& Metabolism. 97(12), 4692-4700.

Myslivecek, P.R., Brown, C.A. \& Wolfe, L.A. (2002). Effects of physical conditioning on cardiac autonomic function in healthy middle-aged women. Journal of Chemical Information and Modeling.27(1), 1-18.

Novais, I.P., Jarrete, A.P., Puga, G.M., Araujo, H.N., Delbin, M.A. \& Zanesco, A. (2017). Effect of aerobic exercise training on cGMP levels and blood pressure in treated hypertensive postmenopausal women. Motriz: Journal of Physical Education.23(1), 1-6.

Puga, G.M., Novais, I. P, Katsanos, C.S. \& Zanesco, A. (2016). Combined effects of aerobic exercise and L-arginine ingestion on blood pressure in normotensive postmenopausal women: A crossover study. Life Sciences. 151, 323-329.

Randolph, J.F., Zheng, H., Sowers, M.R., Crandall, C. Crawford S...Vulga M. (2011). Change in follicle-stimulating hormone and estradiol across the menopausal transition: Effect of age at the final menstrual period. Journal of Clinical Endocrinology \& Metabolism. 96(3), 746754.

Reckelhoff, J.F. (2018). Sex differences in regulation of blood pressure. Advances in Experimental Medicine \& Biology. 1065,139-151.

Rezende, T.M., Sponton, C.H.G., Malagrino, P.A., Bezerra, M.A.C., Penteado, C.F.F. \& Zanesco, A. (2011). Effect of exercise training on the cardiovascular and biochemical parameters in women with eNOS gene polymorphism. Archives of Physiology \& Biochemistry. 117(5), 
265-269.

Sponton, C.H.G., Esposti, R.D., Rodovalho, C.M., Ferreira, M.Jr., Jarrete, A.P...Zanesco, A. (2014). The presence of the NOS3 gene polymorphism for intron 4 mitigates the beneficial effects of exercise training on ambulatory blood pressure monitoring in adults. American Journal of Physiology: Heart and Circulatory Physiology. 306(12), H1679-691.

Sponton, C.H.G., Rezende, T.M., Mallagrino, P.A., Franco-Penteado, C. Bezerra, M.A.\& Zanesco A. (2010). Women with TT genotype for eNOS gene are more responsive in lowering blood pressure in response to exercise. European Journal of Cardiovascular Prevention Rehabilitation. 17(6), 676-681.

Störk, S., van der Schouw, Y.T., Grobbee, D.E. \& Bots, M.L. (2004). Estrogen, inflammation and cardiovascular risk in women: A critical appraisal. Trends in Endocrinology \& Metabolism. $15(2), 66-72$.

Task Force of the European Society of Cardiology and the North American Society of Pacing and Electrophysiology. (1996). Heart rate variability: standards of measurement, physiological interpretation, and clinical use. Task Force of the European Society of Cardiology and the North American Society of Pacing and Electrophysiology. European Heart Journal. 17(3),354-381.

Thompson, D., Karpe, F., Lafontan, M. \& Frayn, K. (2012). Physical activity and exercise in the regulation of human adipose tissue physiology. Physiological Review. 92(1),157-191.

Wang, C-YY., Haskell, W.L., Farrell, S.W., Lamonte, M.J., Blair, S.N...Burt, V.L. (2010). Cardiorespiratory fitness levels among us adults 20-49 years of age: findings from the 19992004 National Health and Nutrition Examination Survey. American Journal of Epidemiology. 171(4), 426-435.

Wassertheil-Smoller, S., Anderson, G., Psaty, B.M., Black, H.R., Manson, J...Lasser, N. (2000). Hypertension and Its Treatment in Postmenopausal Women Baseline Data from the Women's Health Initiative. Hypertension. 36(5),780-789.

Webb, C.M., Ghatei ,M.A., McNeill, J.G. \& Collins, P. (2000). 17ß-Estradiol decreases endothelin-1 levels in the coronary circulation of postmenopausal women with coronary artery disease. Circulation. 102(14), 1617-1622.

Zaros, P.R., Pires, C.E.M.R., Bacci, Jr M., Moraes, C. \& Zanesco. A. (2009). Effect of 6-months of physical exercise on the nitrate/nitrite levels in hypertensive postmenopausal women. 
BMC Women's Health. 9(17), 1-5. 

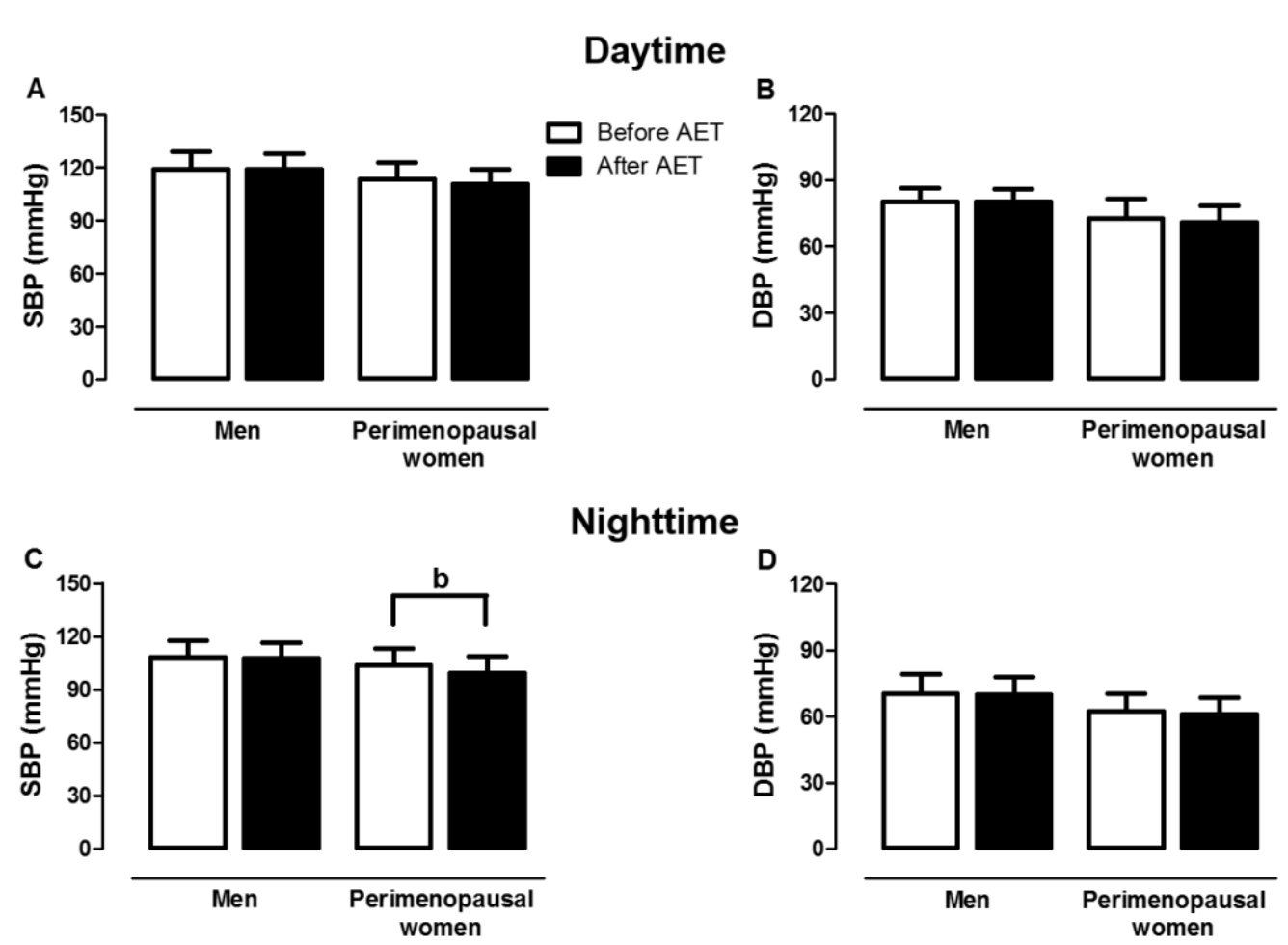

Nighttime
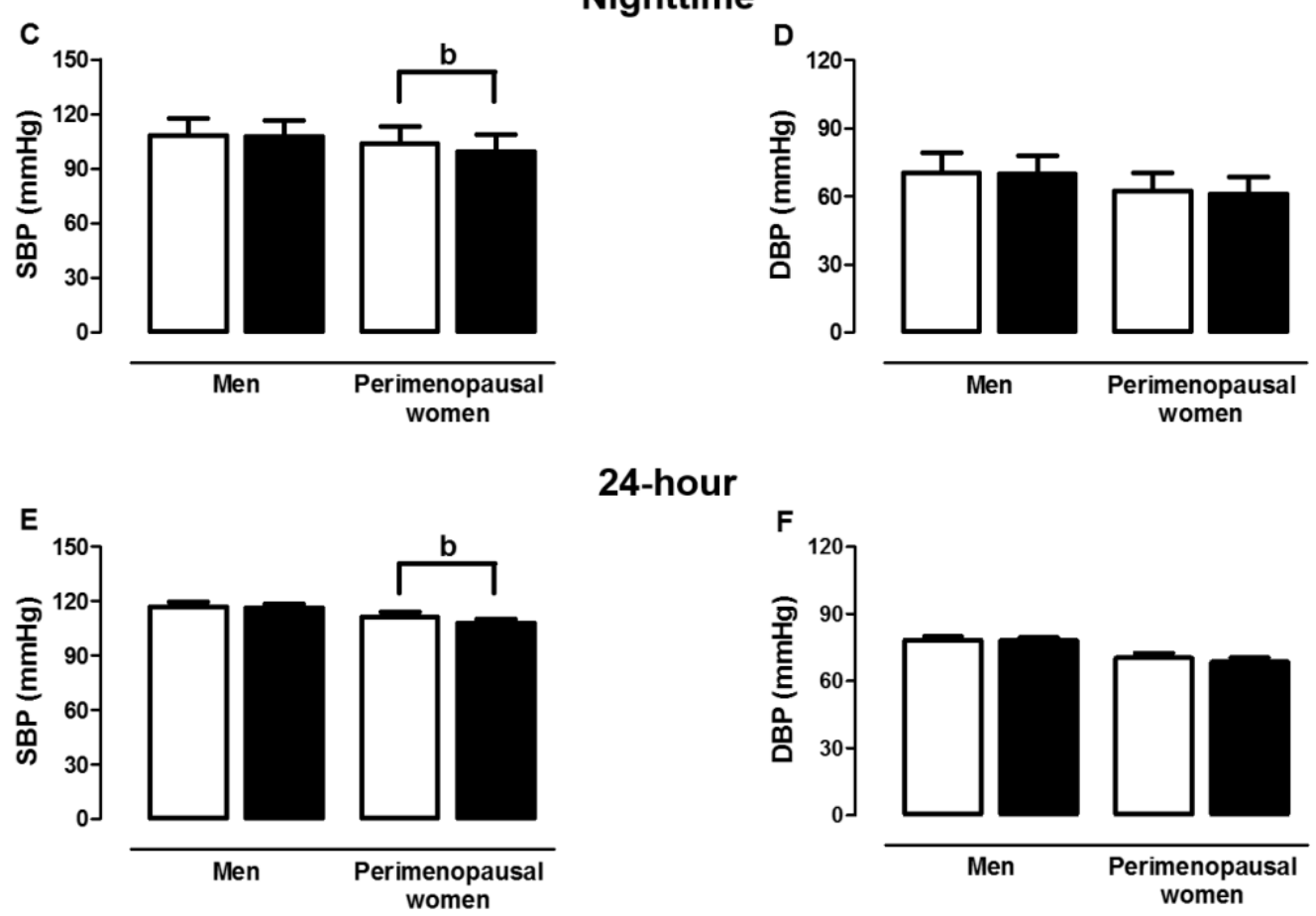

24-hour

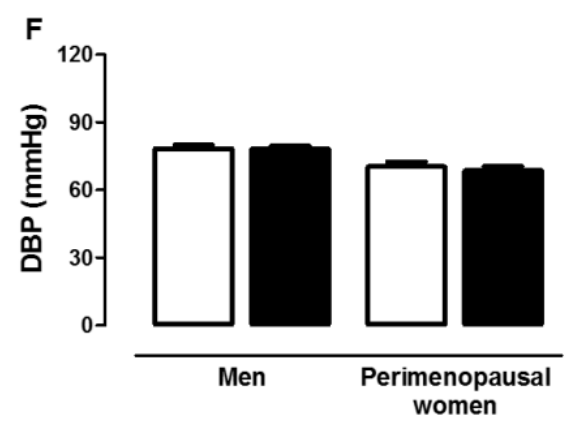

Figure 1. ABPM in perimenopausal women and age-matched men. Panels $A$ to $F$ : Ambulatory blood pressure. $\mathrm{b}=\mathrm{P}<0.05$ compared with before $\mathrm{AET}$ in perimenopausal women. 
Table 1 - The effect of exercise training on anthropometric, cardiovascular and aerobic capacity in perimenopausal won

Parameters

Men $(\mathrm{n}=16)$

Perin

Age, years

Height, cm

Weight, $\mathrm{kg}$

BMI, $\mathrm{kg} / \mathrm{m}^{2}$

Waist circumference, $\mathrm{cm}$

MLSS threshold, $\mu \mathrm{M}$

MLSS exercise HR, beats/min

FSH, $\mathrm{mlU} / \mathrm{mL}$

$\mathrm{LH}, \mathrm{mlU} / \mathrm{mL}$

$17 \beta$-estradiol, pg/ml

Resting HR, beats/min

Peak $\mathrm{O}_{2}$ consumption, $\mathrm{ml} \cdot \mathrm{kg}^{-1} \cdot \mathrm{min}^{-1}$

Office systolic BP, $\mathrm{mmHg}$

Office diastolic BP, mmHg

LF, $\mathrm{ms}^{2}$

$\mathrm{HF}, \mathrm{ms}^{2}$

$\mathrm{LF}, \mathrm{nu}$

$\mathrm{HF}$, nu

Total power, $\mathrm{ms}^{2}$

LF/HF ratio

\begin{tabular}{rrr}
\hline Before AET & After AET & Before AE \\
$173.40 \pm 7.416$ & - & $46.60 \pm$ \\
$80.37 \pm 8.97$ & $79.26 \pm 8.30 \mathrm{a}$ & $161.40 \pm$ \\
$26.95 \pm 2.38$ & $26.60 \pm 2.20 \mathrm{a}$ & $68.59 \pm$ \\
$91.66 \pm 7.76$ & $89.31 \pm 7.05 \mathrm{a}$ & $26.29 \pm$ \\
$3.95 \pm 1.13$ & - & $80.73 \pm$ \\
$158.87 \pm 13.52$ & - & $3.26 \pm$ \\
- & - & $160.64 \pm$ \\
- & - & $19.25 \pm$ \\
- & $69.79 \pm 11.24$ & $19.87 \pm$ \\
$71.21 \pm 7.49$ & $40.71 \pm 2.46 \mathrm{a}$ & $120.57 \pm$ \\
$39.88 \pm 3.14$ & $108.99 \pm 8.17$ & $80.96 \pm 9$. \\
$111.41 \pm 10.89$ & $71.31 \pm 5.80$ & $30.94 \pm 4$. \\
$73.03 \pm 8.36$ & $709.36 \pm 582.18 \mathrm{a}$ & $72.33 \pm 9$. \\
$1509.80 \pm 986.51$ & $385.64 \pm 355.34 \mathrm{a}$ & $699.99 \pm$ \\
$212.37 \pm 193.61$ & $65.47 \pm 14.93 \mathrm{a}$ & $193.18 \pm$ \\
$87.01 \pm 7.70$ & $34.46 \pm 14.89 \mathrm{a}$ & $77.77 \pm$ \\
$12.94 \pm 7.69$ & $2181.90 \pm 1438.81$ & $22.12 \pm$ \\
$2796.82 \pm 1566.05$ & $2.76 \pm 2.37 \mathrm{a}$ & $5.80 \pm$ \\
$10.08 \pm 7.36$ & &
\end{tabular}

Data are mean \pm SD. BMI, body mass index; MLSS, maximal lactate steady state; FSH, follicle-stimulating hormone; L rate; $\mathrm{BP}$, blood pressure; $\mathrm{LF}$, low-frequency components; $\mathrm{HF}$, high frequency components. $\mathrm{a}=\mathrm{P}<0.05$ vs. before AET in perimenopausal women. 